\section{Desmoid Tumor Following Augmentation Mammoplasty with Silicone Implants}

Woo Shik Jeong ${ }^{1}$, Tae Suk Oh${ }^{1}$, Hyung Bo Sim², Jin Sup Eom ${ }^{1}$

${ }^{1}$ Department of Plastic Surgery, Asan Medical Center, University of Ulsan College of Medicine, Seoul; ${ }^{2}$ Plastic Surgery BR, Seoul, Korea

Correspondence: Jin Sup Eom

Department of Plastic Surgery, Asan Medical Center, University of Ulsan College of Medicine, 88 Olympic-ro 43-gil, Songpa-gu, Seoul 138-736, Kore Tel: +82-2-3010-3600, Fax: +82-2-476-7471, E-mail: jinsupp@amc.seoul.kr

This article was presented as a poster at the 70th Congress of the Korean Society of Plastic and Reconstructive Surgeons, November 9-11, 2012 in Seoul, Korea.

No potential conflict of interest relevant to this article was reported.

Received: 27 Feb 2013 • Revised: 24 Mar 2013 • Accepted: 25 Apr 2013 pISSN: 2234-6163・ elSSN: 2234-6171

http://dx.doi.org/10.5999/aps.2013.40.4.470 • Arch Plast Surg 2013;40:470-472

Copyright (C) 2013 The Korean Society of Plastic and Reconstructive Surgeons This is an Open Access article distributed under the terms of the Creative Commons Attribution Non-Commercial License (http://creativecommons.org/licenses/by-nc/3.0/) which permits unrestricted non-commercial use, distribution, and reproduction in any medium, provided the original work is properly cited.

A desmoid tumor (also known as aggressive fibromatosis) is a rare benign lesion that is characterized by fibroblastic proliferation from the connective tissue of the muscle and its overlying aponeurosis of fascia. "Aggressive fibromatosis" is the term used to describe a marked increased in cellularity and aggressive local behavior [1]. Desmoid tumors are characterized by a clinical course that ranges from locally inert to aggressive and destructive forms [2]. Although the mechanisms that affect the development, regulation, and growth of desmoid tumors are currently unknown, numerous factors, such as trauma history, hereditary background (e.g., familial adenomatous polyposis), and the hormonal environment are strongly associated with the development of desmoid tumors $[1,3]$. Desmoid tumors account for $0.03 \%$ of all neoplasms and $0.2 \%$ of all breast tumors. There are few case reports describing implant-associated breast desmoid tumors in the literature [4], and no studies associated with breast implants have been reported in Korea. We report here a case of a desmoid tumor that developed following augmentation mammoplasty and describe our experience of treating this case.

A 34-year-old woman was referred to our hospital in February 2012 due to a painful mass in the upper part of her left breast that was increasing in size. She had undergone augmentation mammoplasty with silicone implants at a local clinic in January 2010, but the implant had been changed because capsular contracture had developed in August 2010. Two years after the implant was changed, this patient's symptoms, including pain and asymmetry, became progressively more aggravated. She visited another local clinic and the implant was scheduled to be changed again to relieve the symptoms of capsular contracture in February 2012. During this surgery, a palpable mass was incidentally found under the pectoralis muscle of her left breast, and she was referred to our hospital.

Preoperative magnetic resonance imaging (MRI) revealed a well-defined solid submuscular mass with low to intermediate signal intensity on $\mathrm{T} 1$ imaging and high signal intensity on $\mathrm{T} 2$ imaging, demonstrating a size of $6.4 \mathrm{~cm} \times 2.6 \mathrm{~cm} \times 5.8 \mathrm{~cm}$ (Fig. 1). Tumor excision and capsulectomy were subsequently performed. The diagnosis of frozen biopsy was benign
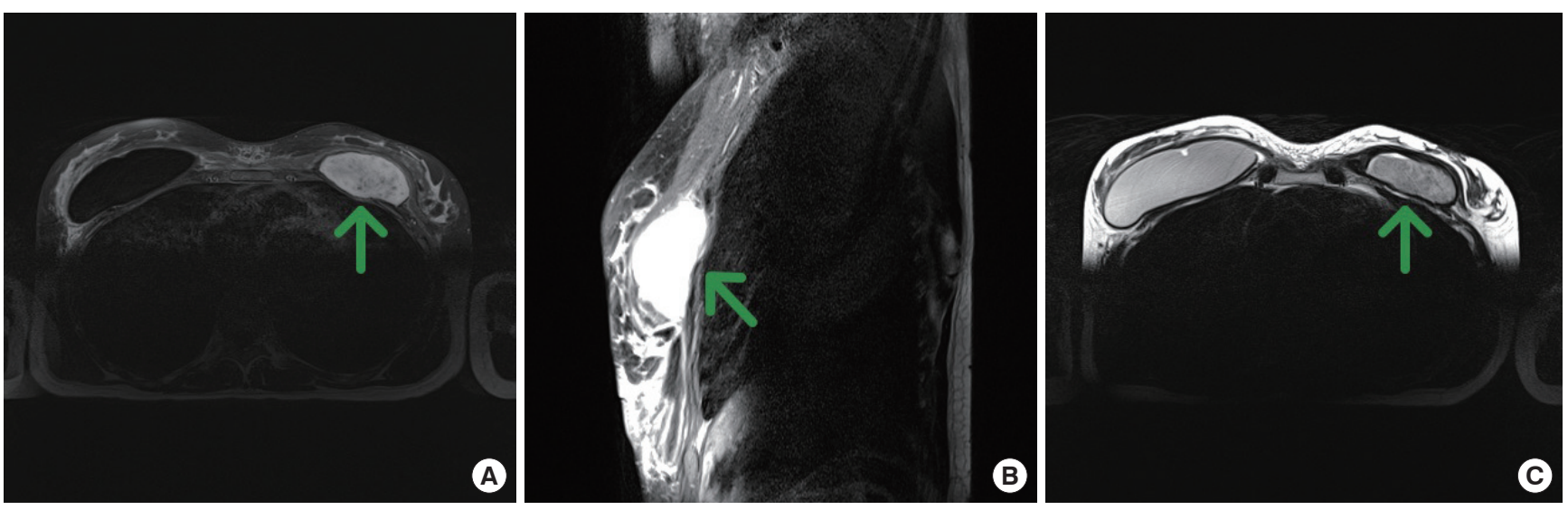

Fig. 1

A well-defined soft tissue mass (green arrow) measuring $6.4 \mathrm{~cm} \times 2.6 \mathrm{~cm} \times 5.8 \mathrm{~cm}$ in the submuscular area of the left anterior chest is shown.

(A), (B) T1 imaging, and (C) T2 imaging. 
mesenchymal tumor, type deferred. Based on frozen biopsy and perioperative findings, we decided to excise as much of the tumor as possible rather than excise widely including normal tissue for a clear resection margin. On gross examination, the tumor was dense and rubbery, and the cut surface was relatively homogeneous demonstrating a fine network of grayishyellow bundles (Fig. 2). After the operation, the final diagnosis of desmoid tumor (fibromatosis) with an indeterminate resection margin was confirmed pathologically (Fig. 3). The patient refused the option of a reexcision procedure and MRI follow-up. She wanted another augmentation mammoplasty, which we refused for oncologic safety, and she was then lost to follow-up.

The natural progression of desmoid tumors remains unpredictable and enigmatic. While this disease rapidly and aggressively progresses in some cases, in other cases, desmoid tumors tend to demonstrate slow and locally invasive growth. Partial regression as well as periods of rapid growth have been reported. However, unlike malignant tumors, these locally invasive tumors do not metastasize. The survival rates are, therefore, excellent, although recurrence and complication rates are high for this disease [5].

Extra-abdominal desmoid tumors are always confined to the connective tissue of the muscle and its overlying aponeurosis of fascia. Thus, when these lesions are found in association with breast implants, the fibrous capsule that forms around the implant is typically implicated as a possible source. Desmoid tumors tend to be hard and firm in consistency, demonstrate a gritty sensation when cut, and have a crosssectional surface that is glistening, white, and coarsely

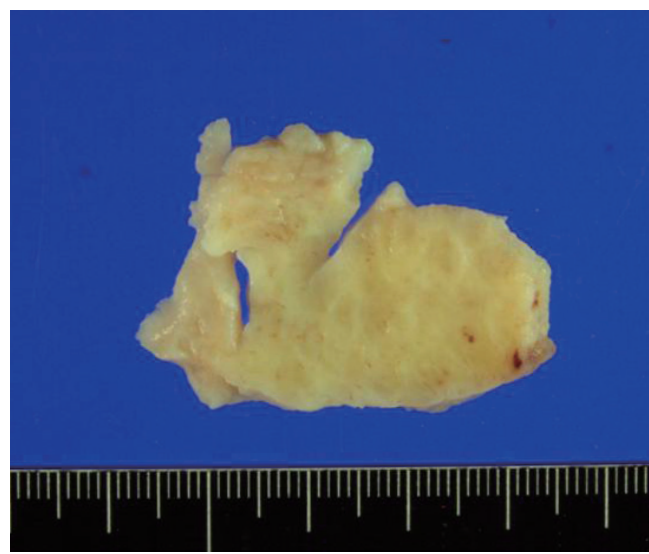

Fig. 2.

Fragmented pathological specimen with grayish-yellow, solid, glistening, and fibrotic cut surface.

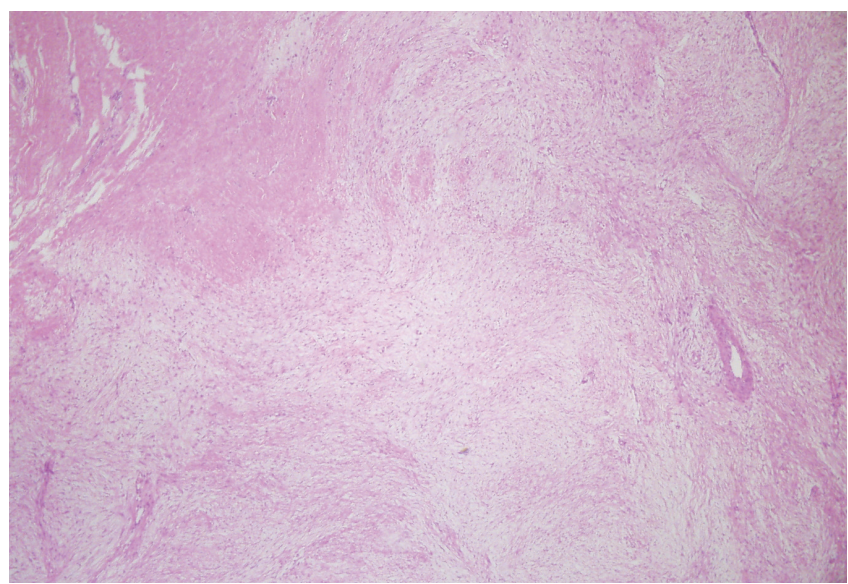

Fig. 3 .

Microscopic image of the pathological specimen. Clusters of spindle-shaped cells and fibroblasts are shown $\left(H \& E_{1} \times 100\right)$.

trabeculated. Histologically, a spectrum of microscopic patterns is often observed, including small clusters or sheets of spindle-shaped cells and fibroblasts, which demonstrate abundant eosinophilic cytoplasm and are separated by large quantities of collagen. Mitotic figures and notable nuclear atypia are rare, although they may be focally abundant with small nuclei that lack pleomorphisms [3]. These characteristic features explain why fine-needle aspiration of these lesions often fails to confirm a diagnosis. Desmoid tumors are typically isointense on T1-weighted imaging and demonstrate low to high signal intensity on T2weighted imaging. MRI analyses in conjunction with the intravenous administration of a contrast agent typically demonstrate heterogeneous gradual enhancement that is believed to reflect a significant amount of collagenous tissue in the tumor. MRI can also play an invaluable role in preoperative diagnosis and planning. The excision of a desmoid tumor with negative margins plays a key role in management and is among the principal therapeutic modalities that are currently available. The treatment aim is usually curative, especially for extra-abdominal, localized, small-volume cases. Local recurrence following surgery can vary considerably depending on the surgical margin status. Radiotherapy, hormonal therapy, and chemotherapy can also be applied, but there are currently no evidencebased pharmacological or radiotherapeutic treatment protocols [4].

Only 24 cases of implant-associated breast desmoid tumors have been described in the literature. Among these, only eight cases developed following the implantation of saline implants. The remaining 16 cases 
developed in association with silicone implants [4]. These lesions may develop after the implantation of either saline or silicone implants, but they develop more commonly when silicone implants are used. When a breast mass has developed after implant insertion, physicians must consider desmoid tumor and anaplastic large cell lymphoma in the diagnosis, although the incidence is low.

This article is the first report describing an implantassociated breast desmoid tumor in Korea. Although there is no evidence to suggest that breast augmentation with either saline or silicone implants is associated with an increased risk of developing desmoid tumors, appropriate vigilance and follow-up examinations are necessary to ensure a timely diagnosis, especially in women who develop capsular contracture following breast augmentation.

\section{References}

1. Shields CJ, Winter DC, Kirwan WO, et al. Desmoid tumours. Eur J Surg Oncol 2001;27:701-6.

2. Chummun S, McLean NR, Abraham S, et al. Desmoid tumour of the breast. J Plast Reconstr Aesthet Surg 2010; 63:339-45

3. Sakorafas GH, Nissotakis C, Peros G. Abdominal desmoid tumors. Surg Oncol 2007;16:131-42.

4. Matrai Z, Toth L, Gulyas G, et al. A desmoid tumor associated with a ruptured silicone breast implant. Plast Reconstr Surg 2011;127:1e-4e.

5. Godwin Y, McCulloch TA, Sully L. Extra-abdominal desmoid tumour of the breast: review of the primary management and the implications for breast reconstruction. Br J Plast Surg 2001;54:268-71.

\section{Mycosis Fungoides Misdiagnosed As Squamous Cell Carcinoma}

Sang Wha Kim, Dong Jin Kim, Sung-No Jung

Department of Plastic and Reconstructive Surgery, Uijongbu St. Mary's Hospital, The Catholic University of Korea College of Medicine, Uijeongbu, Korea

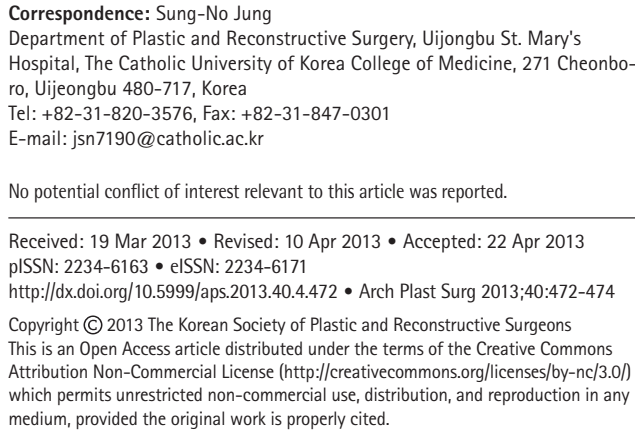

Mycosis fungoides (MF) is the most common type of cutaneous T-cell lymphoma (CTCL) [1]. It is progressive and is associated with poor prognoses in the later stages. The diagnosis of MF remains difficult due to the nonspecificity of the skin lesions and low prevalence. There are broad-based therapeutic options, but the choice of treatment strategy is difficult [2]. Currently, the treatment of MF is divided into two categories: topical therapy, such as corticosteroid, retinoid, and phototherapy, and systemic therapy, such as chemotherapy, electron beam therapy, and radiation therapy [3]. Surgical excision is not considered a first-line treatment option, especially in late-

Preoperative findings of the mass. A 56-year-old man presented with a 2-year history of a solitary and protruding mass on his flank. The lesion was $7 \mathrm{~cm} \times 6 \mathrm{~cm}$ and was erythematous, ulcerated, and bleeding.

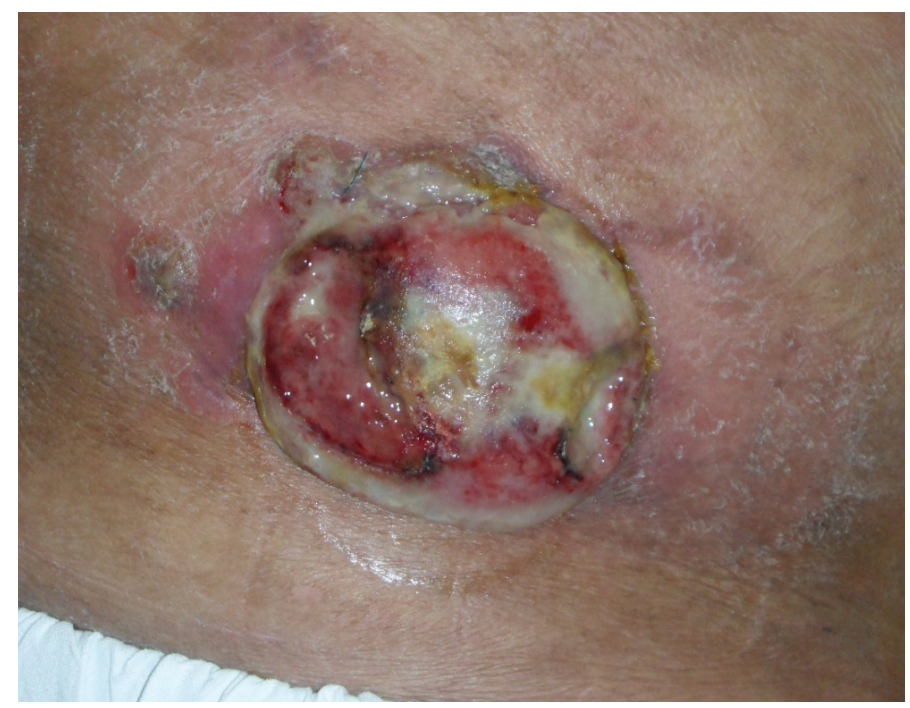

